\title{
Myocardial SPECT Perfusion Defects in Angina Patients, Without Significant Epicardial Coronary Artery Disease. Can Pathological SPECT Affect Long-Term Prognosis?
}

\section{EVANGELOS LAMPAS ( $\nabla$ elampas@hotmail.com )}

Konstantopouleio Geniko Nosokomeio: Konstantopouleio Geniko Nosokomeio Neas Ionias Patesion https://orcid.org/0000-0002-1382-4733

\section{Kiriaki Syrmali}

Konstantopouleio General Hospital Neas Ionias - Agia Olga: Konstantopouleio Geniko Nosokomeio Neas lonias Patesion

\section{Georgios Nikitas}

Panarkadikon Genaral Hospital of Peloponese, Tripoli

\section{Emmanouil C. Papadakis}

Konstantopouleio General Hospital Neas Ionias - Agia Olga: Konstantopouleio Geniko Nosokomeio Neas lonias Patesion

\section{Sotirios P. Patsilinakos}

Konstantopouleio Geniko Nosokomeio: Konstantopouleio Geniko Nosokomeio Neas lonias Patesion

\section{Research Article}

Keywords: False positive SPECT, coronary angiography, cardiovascular morbidity and mortality, revascularization, INOCA

Posted Date: October 22nd, 2021

DOl: https://doi.org/10.21203/rs.3.rs-988779/v1

License: (c) (1) This work is licensed under a Creative Commons Attribution 4.0 International License. Read Full License 


\section{Abstract}

Purpose: Patients with angina and a positive SPECT for reversible ischemia, with no or non-obstructive CAD on ICA represent a frequent clinical problem and predicting prognosis is challenging.

Methods: A retrospective single center study focused on patients that underwent elective-ICA with angina and a positive SECT with no or non-obstructive CAD in the CathLab, during a seven-year period. Assessment of patients' cardiovascular morbidity, mortality, and MACE during a follow-up period of at least three years after ICA, with the assist of a telephone questionnaire.

Results: Data of all patients that underwent ICA for a period of 7 years (from January 1,2011 until December 31,2017$)$ in our hospital were analyzed. The patients that fulfilled the prespecified criteria were 569. At the telephone survey, 285(50.1\%) were successfully contacted and agreed to participate. The mean age was 67.6 (SD8.8) years (35.4\% female) and the mean follow-up time was 5.53years (SD1.85). Mortality rate was $1.7 \%$ (4 patients/non-cardiac causes) and $1,7 \%$ rate of revascularization. $31(10,9 \%)$ were hospitalized for cardiac reasons and $10,9 \%$ patients reported symptoms of HF (no patients with NYHA-Class above II). 21 had arrhythmic events and only two mild anginal symptoms. Noteworthy finding was, the mortality rate in the not-contacted group (12 out of $284,4,2 \%$ ), derived by public security records, did not differ significantly from the contacted-group.

Conclusions: Patients with angina, a positive SPECT for reversible ischemia and no or non-obstructive CAD in ICA have very good long-term cardiovascular prognosis for at least 5 years.

\section{Introduction}

Myocardial perfusion scintigraphy (MPS) imaging has become established as the main functional cardiac imaging technique for ruling-in and ruling-out myocardial ischemia for patients with angina [1]. Thallium stress scintigraphy, either following an exercise test or using pharmacologic stressors was the mainstay for myocardial ischemia diagnosis for decades. Lately technetium and single-photon emission tomography (SPECT) technique have replaced thallium and traditional scintigraphy respectively in the vast majority of studies providing high diagnostic accuracy with low radiation. Defects in stress and rest images of a myocardial SPECT scan are considered indicative of impaired myocardial perfusion, usually due to stenosis or even total occlusion of coronary arteries [2]. The degree and level of epicardial coronary artery stenosis cannot be determined unless angiography is conducted. In angiography "normal" -appearing coronary arteries are defined as having $0 \%$ or $<20 \%$ luminal stenosis and nonobstructive CAD (NOCAD) is defined as coronary arteries with luminal stenosis $>20 \%$ but $<50 \%$. Although traditional understanding of obstructive CAD was $70 \%$, recent European Society of Cardiology and American College of Cardiology/American Heart Association (ACC/AHA) guidelines shifted to include stenosis of $50-70 \%$ if there is associated inducible ischemia or fractional flow reserve $\leq 0.80$ when considering the physiological significance of stenosis and revascularization management in patients with stable CAD [3]. Several studies have shown a particularly high percentage of false positive SPECT 
scans for significant epicardial CAD, ranging from $25 \%$ up to more than $50 \%$ depending on the study, for patients with no or non-obstructive coronary artery disease $[4,5,6]$. Perfusion defects in SPECT scans of these patients may sometimes be artifacts caused by technical issues (attenuation of signal due to radiation absorption from adjacent tissues like the diaphragm being the most common cause) [7]. Yet there is also concern about other pathologies in these patients (such as myocardial "bridges", vasospastic angina, or impaired microvascular flow as in diabetes and hypertensive cardiomyopathylately referred as INOCA) $[3,8,9,10]$ associated with increased cardiovascular morbidity and poor prognosis in the following years. Long-term follow up of patients after a false positive SPECT scan could provide answers to these questions.

\section{Methods}

\section{Study population}

We conducted a retrospective single center study reviewing the database of the Catheterization Laboratory of a tertiary General Hospital carrying out approximately 1600-1700 catheterizations per year. Our initial search focused on patients who underwent invasive coronary angiography with an indication of angina and a positive myocardial SPECT scan for reversible ischemia during a prespecified period. This timeframe was seven years, finishing three years before the retrospective study was conducted, with an intention to extend the minimum follow-up period for every patient included in our study to more than three years. We excluded all patients with significant epicardial coronary artery disease who would proceed to revascularization (either percutaneous or surgical). We also excluded patients with comorbidities which made them unsuitable for intervention and were consulted to restrict to medical treatment, although angiography revealed significant CAD. Finally, due to concerns of a high probability of false positive SPECT results $[11,12]$, all patients with an LBBB morphology in resting ECG were also excluded from the study.

The remaining patients, with no or non-obstructive epicardial CAD, symptoms of angina and a positive myocardial SPECT, were studied further. Telephone contact was attempted with the patients- or their family- and those that agreed to participate to the study answered a structured questionnaire. The questionnaire included questions on cardiovascular morbidity and mortality, targeting mainly to death from any cause, cardiovascular death, hospitalization for any reason related to cardiovascular pathology, myocardial revascularization, angina, arrhythmias, and heart failure. The questionnaire is presented in Table 1.

For patients who could not be reached via telephone contact, we searched in public records using their social security numbers and tracing those who were deceased. This is how we created a "control group" for mortality, since we were concerned about significantly higher percentage of death in patients that could not be contacted. All other data for the deceased patients or patients that could not be contacted were not surveyed at all, since it was impossible to have consent for their inclusion in the study. The 
protocol of the study, as well as the questionnaire used for the telephone interviews, were approved by the Ethics committee of the hospital.

\section{Statistical analysis}

Variables with approximately symmetric distributions were summarized as mean and standard deviation (SD). For comparisons of proportions, chi-square tests were used. All $p$ values reported are two-tailed. Statistical significance was set at 0.05 and analyses were conducted using SOFA-statistics software (version 1.5.3).

\section{Results}

During January 2021 we retrospectively analyzed data of all patients that underwent coronary angiography in the Catheterization Laboratory of the Konstantopouleio General Hospital of Nea lonia for a period of seven years (from January 1,2011 until December 31, 2017). The total number of patients that fulfilled criteria of angina and a positive myocardial SPECT scan for reversible ischemia was 1643 (from a total of 12536 angiographies). No or non-significant coronary artery disease was the diagnosis after coronary angiography in 569 patients (34.63\%). At the telephone survey that followed, $285(50,1 \%)$ of them were successfully contacted, either in person or via a close relative that answered the call and agreed to participate to the study. The flowchart of the study is depicted in Scheme 1. The mean age of patients included in the analysis was 67.6 (SD8.8) years (35.4\% female) and the mean follow up time was 5.53 years (SD1.85). At the time of telephone contact five (5) had died from any cause $(1.7 \%)$, however none of them had died of cardiac causes. Five (1.7\%) underwent revascularization (4 percutaneous and 1 patient coronary artery bypass graft during valve replacement surgery) in the following years, in a mean period of approximately 6 years after the index coronary angiogram, and 6 patients $(2,1 \%)$ had another coronary angiogram during the following years, yet without significant epicardial coronary artery disease and need for revascularization. A much greater number of patients had arrhythmic events in the following years, with a composite of new atrial fibrillation or flutter, ablation for arrhythmias of any origin and pacemaker implantation in 21 patients $(7.4 \%)$. Two patients $(0.7 \%)$ reported symptoms of angina (both CCS class I) and two patients $(0.7 \%)$ had a valve replacement surgery. The total number of hospitalized patients for cardiovascular reasons during follow up was 31 patients (10.9\%), none of them with symptoms of heart failure. Another 31 patients $(10.9 \%)$ reported symptoms of heart failure in the follow up years, but with a mean NYHA class value of only 1.16 and no patients in NYHA class more than II. No patients reported definite symptoms of angina. Another important finding is that the mortality rate in the group of patients that could not be contacted (12 patients out of $284,4,2 \%)$ did not differ significantly from that of the contacted patients [ $X^{2}(1, N=569)$ $=2.07, p=.15]$. These results are depicted in Table 2. Moreover, it is well-established that INOCA is more frequent among women (approximately $50-70 \%$, compared to men) (3). In our study, the subgroup of female patients numbered 101 individuals with a mean age of 69,2, SD8,4 years and a mean follow up time of 5,51, SD1,69 years. Mortality rate was less than 1\% (1 death from non-cardiac reasons, 0,99\%) 
events/interventions and 8 hospitalizations. These results are depicted in Table 3. Finally, another worthreporting result is that all the observed myocardial bridges $(9,3.2 \%)$ were in male patients at the left anterior descending artery course and did not relate to adverse long-term outcomes (except for mild dyspnea in exertion in two of them).

Table 2

Study results.

\begin{tabular}{|ll|}
\hline Number of patients contacted & $\mathbf{2 8 5}$ \\
\hline MeanAge in years (standard deviation) & $67.6(8.8)$ \\
\hline Sex (female) & $101(35.4 \%)$ \\
\hline Dellow up in years after angiography (standard deviation) & $5.53 y r s(1.85)$ \\
\hline Revascularization (PCl/CABG+valve) & $5(1.7 \%)$ \\
\hline Angina (CCS class I) & $4 / 1($ total 1.7\%) \\
\hline New coronary angiography without significant CAD & $2(0.7 \%)$ \\
\hline Hospitalization for cardiovascular reasons & $6(2.1 \%)$ \\
\hline ACS, PCl, Arrhythmic events and ablation, Device implantation) & $31(10.9 \%)$ \\
\hline Arrhythmic events/Interventions for arrhythmia (ablation or device implantation) & $21(7.4 \%)$ \\
\hline Heart failure & $31(10.9 \%)$ \\
\hline (NYHA class-mean) & $(1.16)$ \\
\hline Valve replacement & $2(0.7 \%)$ \\
\hline Number of patients not contacted (data derived from public records) & $\mathbf{2 8 4}$ \\
\hline Deaths & $12(4.2 \%)$ \\
\hline Total Number of patients & $\mathbf{5 6 9}$ \\
\hline Deaths & $17(2,98 \%)$ \\
\hline
\end{tabular}


Table 3

\begin{tabular}{|ll|}
\hline Number of women contacted (101) & \\
\hline Mean Age years (standard deviation) & $69,2(8)$. \\
\hline Follow up years after angiography (standard deviation) & $5.51 \mathrm{yrs}(1.69)$ \\
\hline Deaths (none of them of cardiac origin) & $1(0,99 \%)$ \\
\hline Revascularization (PCI/CABG+valve) & $1(0,99 \%)$ \\
\hline Angina (CCS class I) & $1(0.99 \%)$ \\
\hline Angiography-no serious CAD & $5(1,7 \%)$ \\
\hline Hospitalization for cardiovascular reasons & $8(7.9 \%)$ \\
\hline (ACS, PCl, Arrhythmic events and ablation, Device implantation) & \\
\hline Arrhythmic events/Interventions & $8(7,9 \%)$ \\
\hline NYHA symptoms & $13(12,8 \%)$ I-II class \\
\hline ICA (with no significant CAD, <50\%) & $9(8,9 \%)$ \\
\hline Valve replacement & None \\
\hline
\end{tabular}

\section{Discussion}

In this small retrospective single-center study we showed that patients with angina and a positive SPECT scan indicating reversible ischemia but no significant epicardial coronary artery disease in coronary angiography have very good long-term prognosis in the following years and very low cardiovascular morbidity and mortality. The particularly long average follow-up time of the study, more than 5.5 years, the evidence of no cardiac deaths and a very low rate of revascularization during follow-up period enhance the value of our results. The fact that the rate of death in the "lost to follow-up" patients was not significantly different could be considered as an indice of similarity between the two groups, at least for major outcomes. The rate of hospitalization for cardiovascular reasons during the follow-up period is quite low and the majority is related to arrhythmic events and conduction disturbances rather than acute coronary syndromes. Symptoms of heart failure are reported by one out of ten patients, but with most of them in NYHA class I and no related hospitalizations. The particularly low number of patients complaining about angina is quite striking, although the questionnaire used is not validated and we cannot exclude the possibility of misinterpretation of angina symptoms or heart failure symptoms.

It is undoubtful that our study lacks sufficient data to identify how many of our patients had true dynamic macro- or microvascular impairment, which could explain the positive SPECT scan results, and how many were just "victims" of artifacts of the study. Yet, the very low overall rate of dismal outcomes in the majority of the patients during a long follow up period probably renders such a quest unnecessary. 
Our study had a low total rate of false positive myocardial SPECT scans. Such rates are reported in early studies of MPS performance in the diagnosis of coronary artery disease, with a significant number of

gated SPECT scans and supine and prone acquisitions ${ }^{(13)}$. Yet later "real world" series report significantly higher rates of false positive scans above $50 \%$, raising questions on the accuracy of the method in rulingin patients for coronary artery disease and the subsequent cost of such a strategy. An explanation for our low rate of false positive scans could be the fact that we excluded from analysis all patients that had an LBBB morphology at rest ECG before coronary angiogram. Furthermore, recent literature suggests that this morphology could be a source of increased false positive SPECT scans and subsequently misdiagnosis, leading to unnecessary coronary angiograms ${ }^{(11)}$.

To sum up, our study has numerous limitations. Firstly, the retrospective nature of the study and the lack of clinical information about the patient's thorough medical history when they underwent the index coronary angiography deprives us of data regarding the etiology of the false positive results of SPECT scans. Secondly a considerable number of patients that could not be contacted is an issue that could raise suspicion of a higher mortality/morbidity and cardiovascular events rate in that group. Yet the fact that the data derived from public social security records did not show statistically significant difference as far as mortality is concerned, (irrespective of the etiology) is partially reassuring. Another limitation is that the follow up is via telephone contact with a non-validated questionnaire and the accuracy of answers could be questioned, particularly when quantitative data like NYHA score are assessed. We also had no data regarding the percentage of exercise stress versus vasodilator stress SPECT scans in our sample, known parameters that affect sensitivity and specificity of CAD diagnosis in MPS. Lastly, the limited SPECT scan data regarding the amount and distribution of area at risk in non-revascularized patients, yet with a diagnosis of "positive SPECT", may have contributed to the minuscule cardiovascular morbidity and mortality in the years following the index coronary angiography.

Finally, we observed a very low morbidity and mortality rate in women participants during more than 5 years follow up. This result is not in accord with other INOCA studies. For example, the National Heart, Lung, and Blood Institute-sponsored Women's Ischemia Syndrome Evaluation (WISE) study, depicted that the prognosis of patients with INOCA is far from benign. ${ }^{(14)}$ The small number of women in our study, as well as our inability to determine the reason for false positive SPECT scan results (artifacts, INOCA or others) may be the reasons for this discrepancy with previous data.

\section{Conclusions}

Myocardial perfusion scintigraphy is an important step between clinical assessment and coronary angiography for patients with symptoms of angina. In this small retrospective study, we showed that the long-term prognosis for patients with angina and a positive SPECT scan for reversible myocardial ischemia with no or non-obstructive epicardial coronary artery disease is very good, as far as cardiovascular morbidity and mortality are concerned. We also depicted that the exclusion of patients with an LBBB morphology in resting ECG can improve the diagnostic accuracy of myocardial SPECT 


\section{Abbreviations}

MPS: Myocardial Perfusion Scintigraphy

SPECT: Single-Photon Emission Computed Tomography

LBBB: Left Bundle Branch Block

CAD: Coronary Artery Disease

ECG: Electrocardiogram

HF-NYHA: Heart Failure-New York Heart Association

PCl: Percutaneous Coronary Intervention

CABG: Coronary-Aortic Bypass Surgery

NOCAD: Non- Obstructive Coronary Artery Disease

INOCA: Ischemia but Non-Obstructive Coronary Artery disease

MACE: Major Adverse Cardiac Events

\section{Declarations}

\section{Declaration of Competing Interest}

All authors have no conflicts of interest to declare.

\section{Funding}

This research did not receive any specific grant from funding agencies in the public, commercial, or notfor-profit sectors.

\section{Acknowledgments}

We gratefully acknowledge the help of the staff of the Catheterization Laboratory of Nea lonia, Athens, Greece.

\section{References}

1. Knuuti J, Wijns W, Saraste A et al. 2019 ESC Guidelines for the diagnosis and management of chronic coronary syndromes. Eur Heart J. 2020 Jan 14;41(3): 407-477.doi:10.1093/eurhear tj/ehz425 
2. Underwood SR, Anagnostopoulos C, Cerqueira M et al (2004 Feb) Myocardial perfusion scintigraphy: the evidence. Eur J Nucl Med Mol Imaging 31(2):261-291. doi:10.1007/s00259-003-1344-5

3. Herscovici R, Sedlak T, Wei J, Pepine CJ, Handberg E, Bairey Merz CN (2018) Ischemia and No Obstructive Coronary Artery Disease (INOCA): What Is the Risk? J Am Heart Assoc. Sep 4;7(17): e 008868. doi: 10.1161/JAHA.118.008868. PMID: 30371178

4. Knuuti J, Ballo H, Juarez-Orozco LE et al (2018) The performance of non-invasive tests to rule-in and rule-out significant coronary artery stenosis, in patients with stable angina: a meta-analysis focused on post-test disease probability. Eur Heart J 39:3322-3330. doi:10.1093/eurheartj/ehy267

5. Silva T, Ramos R, Rio R. False positive results of SPECT - we should minimize the unnecessary referrals to invasive coronary angiography. P5336 | BEDSIDE European Cardiology Congress 2013

6. Escandarian R, Reza Razavi M, Safaei S et al. Evaluation of the Correlation Between Myocardial Perfusion Scan Findings and Invasive Coronary Angiography Results. Middle East J Rehabil Health. 2017 April; 4(2): e45216. doi: 10.5812/mejrh.45216

7. Burrell S, MacDonald A (2006) Artifacts and Pitfalls in Myocardial Perfusion Imaging. J Nucl Med Technol 34:193-211. PMID: 17146108

8. Vallejo E, Morales M, Sánchez I, Sánchez G, Alburez JC, Bialostozky D (2005 May-Jun;12(3):318-23) Myocardial perfusion SPECT imaging in patients with myocardial bridging. J Nucl Cardiol. doi: 10.1016/j.nuclcard.2005.01.009. PMID: 15944537

9. Sakata K, lida K, Kudo M, Yoshida H, Doi O (2005 Feb;69(2):171-6) Prognostic value of I-123 metaiodobenzylguanidine imaging in vasospastic angina without significant coronary stenosis. Circ J. doi: 10.1253/circj.69.171. PMID: 15671608

10. Soman P, Dave D, Udelson J et al (2006) Vascular endothelial dysfunction is associated with reversible myocardial perfusion defects in the absence of obstructive coronary artery disease. Journal of Nuclear Cardiology 13(6):756-760. doi:10.1016/j.nuclcard.2006.08.018

11. Higgins JP, Williams G, Nagel JS, Higgins JA (2006 Oct) Left bundle-branch block artifact on single photon emission computed tomography with technetium Tc $99 \mathrm{~m}(\mathrm{Tc}-99 \mathrm{~m})$ agents: mechanisms and a method to decrease false-positive interpretations. Am Heart J 152(4):619-626. doi:10.1016/j.ahj.2006.06.009

12. Koepfli P, Wyss CA, Gaemperli O, Siegrist PT, Klainguti M, Schepis T, Namdar M, Bechir M, Hoefflinghaus T, Duru F, Kaufmann PA (2009 Dec) Left bundle branch block causes relative but not absolute septal underperfusion during exercise. Eur Heart J 30(24):2993-2999. doi:10.1093/eurheartj/ehp372

13. Berman DS, Kang X, Nishina $\mathrm{H}$ et al (2006) Diagnostic accuracy of gated Tc-99m sestamibi stress myocardial perfusion SPECT with combined supine and prone acquisitions to detect coronary artery disease in obese and nonobese patients. Journal of Nuclear Cardiology 13(2):191-201. doi:10.1007/BF02971243

14. Kunadian V, Chieffo A, Paolo G, Camici et al An EAPCI Expert Consensus Document on Ischaemia Loading [MathJax]/jax/output/CommonHTML/fonts/TeX/fontdata.js on with European Society of Cardiology Working 
Group on Coronary Pathophysiology \& Microcirculation Endorsed by Coronary Vasomotor Disorders International Study Group (October 2020) Eur Heart J 41:3504-3520.

https://doi.org/10.1093/eurheartj/ehaa503. Issue 37, 1

\section{Tables}

Due to technical limitations, table 1 is only available as a download in the Supplemental Files section.

\section{Supplementary Files}

This is a list of supplementary files associated with this preprint. Click to download.

- Scheme01.png

- Table1.docx 